\title{
Topic Study Group No. 17: Teaching and Learning of Discrete Mathematics
}

\author{
Eric W. Hart, James Sandefur, Cecile O. Buffet, \\ Hans-Wolfgang Henn and Ahmed Semri
}

\section{The Programme}

Discrete mathematics is a comparatively young branch of mathematics with no agreed-upon definition but with old roots and emblematic problems. It is a robust field with applications to a variety of real world situations, and as such takes on growing importance in contemporary society.

We take discrete mathematics to include a wide range of topics, including logic, game theory, algorithms, graph theory, discrete geometry, number theory, discrete dynamical systems, fair division, cryptography, coding theory, and counting. Cross-cutting themes include discrete mathematical modeling, algorithmic problem solving, optimization, combinatorial reasoning, and recursive thinking.

Discrete mathematics is not always clearly delimited in curricula and can be diffuse. In fact, two separated but linked curricular perspectives emerge: teaching and learning discrete mathematics content and teaching and learning skills of mathematical practice through discrete mathematics problems, both general skills, such as reasoning and modeling, and skills particular to discrete mathematics, such as algorithmic and recursive thinking. Thus, discrete mathematics provides a useful setting in which to pursue the ongoing problem in mathematics education of the didactic transposition of content knowledge and process skill, and it provides an

Co-chairs: Eric W. Hart, Cecile O. Buffet.

Team members: Hans-Wolfgang Henn, James Sandefur, Ahmed Semri.

E.W. Hart $(\bowtie)$

Georgetown University, Washington, D.C., USA

e-mail: ehart@infinitemath.com; ehart@grandview.edu

C.O. Buffet

Université Reims Champagne Ardenne, Reims, France

(C) The Author(s) 2017

G. Kaiser (ed.), Proceedings of the 13th International Congress on Mathematical

Education, ICME-13 Monographs, DOI 10.1007/978-3-319-62597-3_44 
opportunity to develop and refine models for teaching and learning that develop both.

The main goal of the TSG is to discuss and extend the state-of-the-art about teaching and learning discrete mathematics. The broad focus areas related to this goal are teaching and learning discrete mathematics at all grade levels, research, curriculum development, professional development of teachers, and curricular implementation of discrete mathematics, including policy and standards. Papers from scholars around the world were presented in seven sessions. These papers are briefly summarized below.

\section{Invited Papers}

- Margaret Cozzens, Rutgers University — “Food Webs, Graphs, and a 60-Year Old Problem Students Can Help Solve." Food webs describe the flow of energy through an ecosystem. Middle and high school students encounter food webs in their biology classes. The discrete mathematics related to food webs is usually not discussed in these classes, yet it is relatively easy mathematics that teachers can understand. This paper models food webs with directed graphs, and discusses why competition graphs derived from real food webs seem to be interval graphs.

- Robert Devaney, Boston University - "Discrete Dynamical Systems: A Pathway for Students to Become Enchanted with Mathematics." In this paper we show how the topic from discrete dynamical systems known as the chaos game can be used to get students excited about mathematics. In addition, we describe a number of different ways this topic relates to the standard high school mathematics curriculum.

- Susanna Epp, DePaul University-“Discrete Mathematics for Computer Science." This paper explores some of the issues involved in teaching a discrete mathematics course for computer science students. This paper discusses some of the challenges involved in implementing the recommendations for discrete mathematics instruction published by the computer science societies, explores some of the reasons behind them, and suggests considerations that educators should take into account when they prepare instructional materials.

- Solomon Garfunkel, COMAP_- "Fairness." For the past 45 years I have worked to bring mathematical modeling and applications of mathematics into the mainstream mathematics curricula at all grade levels. This work has continuously (pun intended) bucked up against those who believe that analysis is mathematics and therefore courseware must be designed to prepare students for continuous mathematics. And even those who give a nod to modeling see it in terms of physics and engineering, reinforcing their belief in the calculus escalator. Discrete mathematics also is important. For example, models of fair division are discussed in this paper. 
- Gerald Alan Goldin, Rutgers University - "Discrete Mathematics and the Affective Dimension of Mathematical Learning and Engagement." Discrete mathematics offers some specific affordances for encouraging students to experience mathematics in ways very different from more traditional school subjects such as arithmetic, algebra, geometry, and analysis. Opportunities abound for teachers and curriculum planners to focus on evoking interest and engagement, and on developing powerful affect-emotions, attitudes, beliefs, and values - in relation to mathematics. This paper explores such opportunities in relation to research constructs in the literature on mathematical affect.

- Eric W. Hart, Grand View University - "Discrete Mathematical Modeling in the Secondary Curriculum." In this paper we describe a multistage process of discrete mathematical modeling that is based on many years of curriculum research and development. Five broad problem structures emerge as ways to organize the diversity of discrete mathematics contexts that are important and appropriate for high school-enumeration, sequential change, relationships among a finite number of objects, information processing, and fair decision-making. The process of discrete mathematical modeling is outlined for these five problem structures.

\section{Contributed Papers}

- Tom Coenen, University of Twente-“Combinatorial Reasoning to Solve Problems." This study reports on the combinatorial reasoning of students aged 14-16. We study the variation of the students' problem solving strategies in the context of emergent modeling. The results show that the students are tempted to begin the problem solving process on the highest level and otherwise have difficulties transitioning from a lower to a higher level of activities. We advocate matching emergent modeling with teaching combinatorial reasoning, stimulating students to create a relational network of knowledge.

- Aaron Gaio, University of Palermo - “I Like Discrete Mathematics, But I Do Not Know How To Teach It." The paper describes a research project aiming at bringing new mathematical knowledge and competences to students and involving teachers in the activity designing process. In this context, we present an overview of the Italian situation in teaching discrete mathematics in primary and middle school, together with reference to the national teaching guidelines. We then briefly describe the results obtained from our first survey of about 100 teachers.

- Karina Höveler, TU Dortmund - “Children's Combinatorial Counting Strategies and Their Relationship to Mathematical Counting Principles." This paper reports about selected findings from a qualitative study with third graders. The study's main goals were to identify how children solve combinatorial problems and to gain insights into the relationship between their strategies and mathematical ideas. 
- Vladimir Igoshin, Saratov State University - "Mathematics and Logic: Their Relationship in the Training of Teachers of Mathematics." In the process of teaching and learning mathematics logic cannot be avoided, mathematics and logic prove to be inseparable and interact closely. This interaction has didactic and training implications. After analyzing different aspects of this interaction, the author identifies and substantiates the principles of logic in mathematics and in the education of teachers of mathematics.

- Elise Lockwood, Oregon State University-“Generalization in Students' Combinatorial Thinking." The purpose of this paper is to characterize and better understand the role of generalizing activity in students' combinatorial problem solving. We do this by drawing upon Lockwood's model of combinatorial thinking. The findings come from a series of interviews in which students solved combinatorial tasks designed to foster generalization.

- Maria Flavia Mammana, University of Catania - “Graph Theory in Primary, Middle, and High School.” In this paper we present an experimental teaching activity with topics of graph theory conduced in some primary, middle, and high schools in Sicily. The aim of the whole project is to present a fun, easy approach to mathematics and some connection of mathematics with real life, in order to reach competencies related to the use of mathematical models to solve problems.

- Lisa Rougetet, University of Lille_- "Machines Designed to Play NIM Games as Teaching Support for Mathematics, Algorithmics, and Computer Science (1940-1970)." This paper deals with Nim games and machines that were designed to play against a human between the 1940s and the 1970s. We focus on machines that were intended not only to play, but also to explain concepts in mathematics, algorithmics, and computer science.

- James Sandefur, Georgetown University- "Recursion versus Closed Formulas." This paper promotes the use of recursion and difference equations as a means for promoting mathematical understanding and communication through the use of contextual problems. Specifically, three contextual examples are given, with closed form and recursive solutions to the problems being contrasted.

- Ödön Vancsó, Eötvös Loránd Tudományegyetem-“Complex Mathematics Education in the 21st Century: Improving Combinatorial Thinking Based on T. Varga's Heritage." This paper summarizes the ideas and background of a combinatorics research and teaching project including historical reforms in school curriculum in 1978 in Hungary and T. Varga's work. Thereafter we collect the main elements of our project: pretest and developed teaching materials, worksheets with some examples and showing tools for teaching combinatorics such as Poliuniversum.

- Catherine Vistro-Yu, Ateneo de Manila University- "Discrete Mathematics in the General Education Curriculum." This paper describes some key issues that Filipino mathematics educators face as a new mathematics course is developed for inclusion in the new General Education Curriculum for all higher education institutions in the Philippines. The new course is largely a discrete mathematics course, a sample syllabus of which was designed by the first author. 


\section{Posters}

- Antonio Kennedy Lopes Dantas, Federal Institute of Piauí-“"Clothing and the Use of Hanoi Tower: A Learning in Practice." The work presents the construction of the game Tower of Hanoi as a differentiated education episode that allows students to be active in the construction of knowledge, overcoming with motivation and fun, using content like exponential functions, geometric progression, counting, and geometry. It was conducted with 40 students of the 2nd year of high school, in college Zacarias de Gois, in Teresina, Piauí.

\section{TSG Discussion Group}

The Discrete Mathematics TSG coordinated with a Graph Theory Discussion Group organized by James Maltas. The TSG concluded with an open discussion forum on: "Discrete Mathematics in Standards, Curricula, Classrooms, and Research around the World: Current Issues and Next Steps."

Open Access Except where otherwise noted, this chapter is licensed under a Creative Commons Attribution 4.0 International License. To view a copy of this license, visit http://creativecommons. org/licenses/by/4.0/.

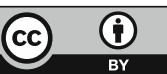

